\title{
Mitteilungen des Bundesverbandes Ärztlicher Leiter Rettungsdienst
}

Bundesverband

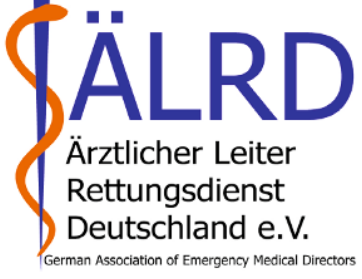

Bundesverband Ärztlicher Leiter Rettungsdienst e.V. Geschäftsstelle

Friedberger Str. 31, 61169 Friedberg

\section{Redaktion:}

Prof. Dr. Dr. A. Lechleuthner (Vorsitzender BV ÄLRD, V.i.S. d.P.)

Jutta Schürmann-Lipsch (ÄLRD Märkischer Kreis)

\section{Herbsttagung des Bundesverbandes ÄLRD e.V.}

\section{Hamburg, 18.09.2017 \\ Wie kann E-Learning zur Fortbildung von Notfallsa- nitätern etabliert werden, wie gehen Rettungsdienste mit hohem Aggressionspo- tential um, wie gelingt die internationale Vernetzung des Rettungsdienstes?}

Zur Diskussion dieser und weiterer aktueller Fragen reisten 80 Teilnehmer zur Herbsttagung des Bundesverbandes ÄLRD e. V. nach Hamburg (• Abb. 1).

Die zweitägige Tagung eröffnete der Vorsitzende des Bundesverbandes der Ärztlichen Leiter Rettungsdienst Professor Dr. Alex Lechleuthner aus Köln. Der Sachverständigenrat zur Begutachtung der Entwicklung im Gesundheitswesen stellte am 07.09.2017 in Berlin Grundzüge seines Gutachtens zur Zukunft der Notfallversorgung dar. Nach Einschätzung des Vorsitzenden stehen in den kommenden Jahren große Veränderungen in der Notfallversorgung an, die auch den Rettungsdienst erheblich verändern werden.

Bereits am Vortag trafen sich die Vertreter der Bundesländer zum Austausch der landesspezifischen Themen. Hierbei zeigte sich eine große Bandbreite der
Herausforderungen die neben den unterschiedlichen Ständen der Notfallsanitäter-Ausbildung und deren Integration in die Notfallversorgung auch den Bereich der arztbegleiteten Verlegungstransporte und den Aufbau von Qualitätsmanagement-Strukturen. Erstmals seit Jahren war der „Notarzt-Mangel“ kein dominierendes Thema. Den Mitgliedern des Bundesverbandes wurden die Länder-Berichte in komprimierter Form dargestellt und gemeinsam diskutiert.

In den Ländern Bayern, Berlin und Schleswig-Holstein sind neue Rettungsdienst-Gesetze in Kraft getreten, in denen die Delegation von heilkundlichen Maßnahmen an Notfallsanitäter durch die Ärztliche Leitung Rettungsdienst geregelt wird.

Die ÄLRD sind derzeit einerseits erheblich in der Gestaltung des Rettungsdienstes gefordert, andererseits aber aus Sicht des Bundesverbandes in vielen Gremien noch nicht ausreichend repräsentiert - allerdings sind die Nachfragen nach Beteiligung steigend.

In einem Fachvortrag stellte Jens Büttner, ÄLRD in Darmstadt, ein E-Learning-Konzept vor, das in der Region Darmstadt-Dieburg zur Fortbildung

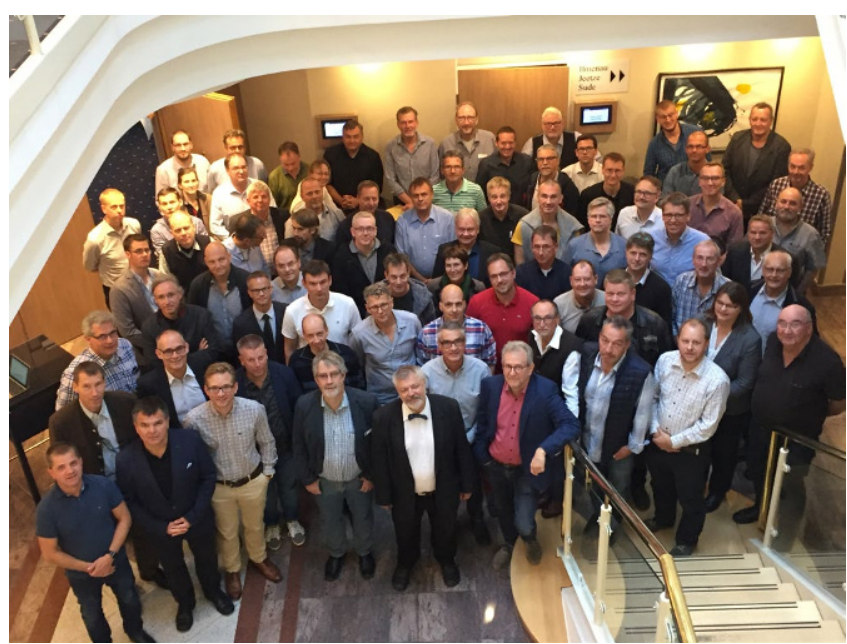

Abb. 1 A Gruppenbild der Teilnehmer der Herbsttagung 2017

des gesamten Rettungsdienstpersonals (inkl. Notärzte) verwendet wird. Gewalt gegen Einsatzkräfte machte Guido Scherer, ÄLRD in Mainz, zum Thema seines Fachvortrags und stellte Initiativen zum Umgang mit diesem Problem bzw. zu Fortbildung des Personals vor.

In vier parallel stattfindenden Arbeitsgruppen wurden Aufgaben des Bundesverbandes erarbeitet. Hierbei ging es um die Entwicklungen im Bereich der Leitstelle, sowie die Nutzung von Medikamenten im Rettungsdienst im Sinne des „offlabel-use“, d.h. jenseits der zu- gelassenen Indikation oder des vorgesehenen Applikationsweges. Weitere Arbeitsgruppen bearbeiteten rechtliche Fragen, insbesondere bei der Ablehnung von Notfallbehandlungen und -transporten und die Neugestaltung der Verbands-Homepage.

Am zweiten Tag wurden die Ergebnisse der Arbeitsgruppen vorgestellt. Es folgten Fachvorträge von Dr. Holger Löning (ÄLRD Flensburg), der ein Tool zur Berechnung des Stellenanteils des ÄLRD vorstellte. Anschließend berichtete Stefan Poloczek aus Berlin über den RettungsdienstKongress in Kopenhagen. 